\title{
Reliability analysis of tensegrity towers in a system approach
}

\author{
W. Mochocki \& P. Obara \\ Kielce University of Technology, Kielce, Poland
}

\begin{abstract}
In this paper, a reliability analysis of tensegrity towers is presented. The towers built with Simplex modules are considered. The analysis of tensegrity structures consists of three stages. The first stage leads to the identification of the existing mechanisms and self-stress states in the structure. The second stage focuses on the determination of reliability models, whereas third - contains determination the influence of the level of self-stress state on static parameters and consequently on the reliability index.
\end{abstract}

\section{INTRODUCTION}

Tensegrities are the structures composed of only compressed (struts) and tensioned elements (cables). A characteristic feature of tensegrity structures is system of internal forces holds the structural components in stable equilibrium. This is called the self-stress state. The changes of the level of self-stress state influences on behaviour of structures, whereas the absence causes that structures are geometrically variable (infinitesimal mechanism occurs). Initially, the idea of tensegrity construction was associated primarily art. Currently, the use of such structures is increasing in civil engineering and tensegrity concept is used among others in towers (Schlaich 2004, Snelson 2013, Obara 2019).

The paper develops the considerations of tensegrity towers presented in (Obara 2019). A new problem concerning on reliability analysis of such structures has been studied. The towers built with Simplex modules (Fuller 1962, Emmerich 1964, Snelson 1965) are considered. The reliability assessment consists in comparing the reliability index of the structure with the required value of the reliability index. The required value of the reliability index was chosen for the RC2 reliability class (50 years reference period). In accordance with the standard (PN-EN 1990:2004), "Three reliability classes $(\mathrm{RC} 1, \mathrm{RC} 2$, and $\mathrm{RC} 3$ ) may be associated with the three consequence classes CC1, CC2, and CC3". The tower's construction was classified as a structure with the consequence class $\mathrm{CC} 2$. This means that "Medium consequence for loss of human life and economic, social, or environmental consequences are considerable". The required value for the reliability class $\mathrm{RC} 2$ is $\beta=3.8$. In this paper the towers built with Simplex modules are considered. The influence of the level of self-stress state on static parameters and the reliability index is analysed. The static analysis is carried assuming the hypothesis of large displacements. In turn to assessing the reliability of a structure the system approach is used wherein the reliability index is calculated according to the assumption of serial connections.

\section{MATHEMATICAL DESCRIPTION}

Reliability analysis of tensegrity structures is a three-stage process. The first stage (the qualitative analysis) includes the identification of self-stress states and infinitesimal mechanisms. The second stage focuses on the determination of reliability models, whereas third - contains 
determination the reliability index in order to the self-stress state. The last two stages are the quantitative analysis.

The first stage is carried out based using singular value decomposition of the compatibility matrix (Pellegrino 1993, Gilewski et al. 2015, 2019, Obara 2019, Obara \& Tomasik 2020). The compatibility matrix for structures is determined using the finite element formalism (Zienkiewicz 2000, Obara \& Tomasik 2020). Tensegrity towers aren-element space trusses $(e=1,2, \ldots, n)$ described by the elasticity matrix $\mathbf{E}\left(\in \mathbb{R}^{n \times n}\right)$ with $m$-degrees of freedom $\mathbf{q}\left(\in \mathbb{R}^{m \times 1}\right)$. The singular value decomposition of matrix $\mathbf{B}\left(\in \mathbb{R}^{n}\right)$ is a factorization in the form:

$$
\mathbf{B}=\mathbf{Y} \mathbf{N X}^{T},
$$

where $\mathbf{Y}\left(\in \mathbb{R}^{n \times n}\right)=\left[\begin{array}{llll}\mathbf{y}_{1} & \mathbf{y}_{2} & \ldots & \mathbf{y}_{n}\end{array}\right]$ and $\mathbf{X}\left(\in \mathbb{R}^{m \times m}\right)=\left[\begin{array}{llll}\mathbf{x}_{1} & \mathbf{x}_{2} & \ldots & \mathbf{x}_{m}\end{array}\right]$ are orthogonal matrices and $\mathbf{N}\left(\in \mathbb{R}^{n \times m}\right)$ is a rectangular diagonal matrix. The orthogonal matrices $\mathbf{Y}$ and $\mathbf{X}$ as well as matrix $\mathbf{N}$ are related to eigenvectors and eigenvalues of the following problems:

$$
\left(\mathbf{B B}^{T}-\mu \mathbf{I}\right) \mathbf{y}=\mathbf{0},\left(\mathbf{B}^{T} \mathbf{B}-\lambda \mathbf{I}\right) \mathbf{x}=\mathbf{0},
$$

where $\mu$ and $\lambda$ are eigenvalues of the respective matrix. If any $\mu$ value is equal to zero, a selfstress state exists. If any $\lambda$ value is equal to zero, a mechanism exists. In order to identify whether the mechanism is infinitesimal or finite, nonlinear analysis of the stiffness matrix with the use of geometric stiffness matrix $\mathbf{K}_{G}(S)$, which takes into account the self-stress state $S$, should be applied. If all eigenvalues of the matrix $\left(\mathbf{B}^{T} \mathbf{E B}+\mathbf{K}_{G}(S)\right)$ are positive, the identified mechanism is infinitesimal and the structure is stable.

In the second stage a reliability model must be defined. Due to all analysed towers are tensegrities, which are characterized by mechanisms occurs, a reliability model is a serial system. Reliability of the system $R$ is calculated from the formula:

$$
R=\prod_{i=1}^{n} R_{i}
$$

where $R_{i}$ is reliability of a single element. The procedure that leads to the determination of the reliability of element $R_{i}$ was presented, among others, in papers (Mochocki et al. 2018, 2019, 2020). To do this, the static analysis must be performed. It is necessary to determine random variables of the effect of action $E_{i}$, and of capacity $N_{i}$. Random variables are described by means of the normal distribution with the parameters expected value $\mu$ and standard deviation $\sigma$. With respect to standard deviation for effect of actions $E_{i}$, the variation coefficient is assumed at the level of $6 \%$, whereas with standard deviation for capacity $N_{i}$, it is at the level of $10 \%$. A subsequent step is determining the safety margin $Z_{i}$, which is also a random variable described by means of the normal distribution. The parameters of this system are the expected value of the safety margin $\mu_{Z i}=\mu_{N i}-\mu_{E i}$ and standard deviation of the safety margin $\sigma_{Z i}=\left(\sigma_{E i}^{2}+\sigma_{N i}^{2}\right)^{0.5}$. Next, the reliability index for a single element is computed $\beta_{i}=\mu_{Z i} / \sigma_{Z i}$. On its basis, the probability of the element failure can be estimated $P_{f i}=\Phi\left(-\beta_{i}\right)$, where $\Phi$ is the Laplace function. The final step involves the computation of the reliability of a single element $R_{i}=1-P_{f i}$ and at the end - the reliability of the system $R(3)$.

In the last, third stage, the reliability index is calculated:

$$
\beta=-\Phi^{-1}(1-R) .
$$

It must be added, in the case of tensegrity structures the static parameters depend on the level of self-stress state. It means, the self-stress state consequently influences on the reliability index (4). 
In order to fully understand the behaviour of such structures, in the first the static analysis was carried out. The influence of the level of self-stress state on the normal forces $N$, the loadbearing capacity ratio $W_{\max }=N / N_{R d}$ and the stiffness were calculated. The static analysis was carried out assuming the hypothesis of large displacements:

$$
\mathbf{K}_{S} \mathbf{q}=\mathbf{P} ; \mathbf{K}_{S}=\left[\mathbf{B}^{T} \mathbf{E B}+\mathbf{K}_{G}(N+S)+\mathbf{K}_{N, N L}(\mathbf{q})\right],
$$

where $\mathbf{P}$ is the load vector, $\mathbf{K}_{G}(N+S)$ is the geometric stiffness matrix, which takes into account the self-stress state $S$ and the normal force $N$ and $\mathbf{K}_{N, N L}(\mathbf{q})$ is the initial strain matrix. The change in rigidity was measured using the so-called global stiffness parameter (GPS) (Obara 2019). The GPS parameter expresses the ratio of two strain energies, measured at the minimum and at the $i$-th level of self-stress:

$$
G P S=\frac{\left[\mathbf{q}\left(S_{\min }\right)\right]^{T} \mathbf{K}_{S}\left(S_{\min }\right) \mathbf{q}\left(S_{\min }\right)}{\left[\mathbf{q}\left(S_{i}\right)\right]^{T} \mathbf{K}_{S}\left(S_{i}\right) \mathbf{q}\left(S_{i}\right)},
$$

where $\mathbf{K}_{S}\left(S_{\text {min }}\right)$ and $\mathbf{q}\left(S_{\text {min }}\right)$ are a secant stiffness matrix and a design displacement vector with a minimum level of self-stress state, and $\mathbf{K}_{S}\left(S_{i}\right)$ and $\mathbf{q}\left(S_{i}\right)$ - at $i$-th level of self-stress state.

\section{EXAMPLES}

In this paper, the reliability analysis of tensegrity towers is performed. As the first, qualitative analysis leads to classification towers to tensegrity groups are carried out. Next, the calculations of the influence of self-stress state on the normal forces, the load-bearing capacity ratio, the stiffness and, at the end, on the reliability index are performed. The structures built with Simplex modules are taken into account. The single module is considered as the first and then towers build with two and six modules are analysed. The design solution of the Halfen DETAN Rod System is adopted and the following characteristics are assumed:

- Young modulus: $E=210 \mathrm{GPa}$ and density: $\rho=7860 \mathrm{~kg} / \mathrm{m}^{3}$

- cables: made of rods, steel S460N, diameter $\phi=20 \mathrm{~mm}$, moment of inertia: $I=7.85 \cdot 10^{9} \mathrm{~m}^{4}$, cross-sectional area: $A=3.14 \cdot 10^{4} \mathrm{~m}^{2}$, load-bearing capacity: $N_{R d}=110.2 \mathrm{kN}$,

- struts: made of hot-finished circular hollow section, steel: S355J2, diameter: $\phi=76.1 \mathrm{~mm}$, thickness: $t=2.9 \mathrm{~mm}$, moment of inertia: $I=4.47 \cdot 10^{7} \mathrm{~m}^{4}$, cross-sectional area: $A=6.88 \cdot 10^{4} \mathrm{~m}^{2}$, load-bearing capacity: $N_{R d}=203.5 \mathrm{kN}$.

Normalized self-stress state forces were multiplied by the level of self-stress state $S$ and applied to the structure. The minimum applied level of the self-stress state $S_{\min }$ provides proper identification of the elements (i.e. struts are compressed and cables are tensed). The maximum level of the self-stress state $S_{\max }$ does not cause the exceedance of the load-bearing capacity of the elements. These levels are equal respectively: $S_{\min }=1 \mathrm{kN}$ and $S_{\max }=110 \mathrm{kN}$. The models was loaded with a vertical force $P=-10 \mathrm{kN}$ and $P=-20 \mathrm{kN}$ applied to one top node. For calculation, a procedure in the "Mathematica" environment was created.

\subsection{Qualitative analysis}

The first considered structure is the single Simplex module (model S1) (Figure 1a), which consists of twenty elements $(n=12)$, i.e. three struts and nine cables, and six nodes. The coordinates of the nodes are shown in Table 1 . The analysis was performed for the module with twelve degrees of freedom $(m=12)$ (the blocked displacements are $\left.q_{1}, q_{3}, q_{5}, q_{6}, q_{7}, q_{9}\right)$ thus the number of elements and the number of degrees of freedom are equal $(n=m=12)$. The 
a)

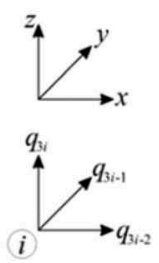

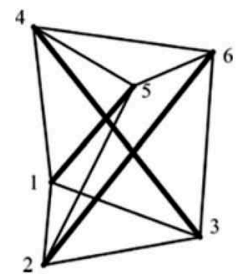

b) struts:

$-1.0000$

top and bottom cables:

0.3853

middle cables:

0.6966 c)

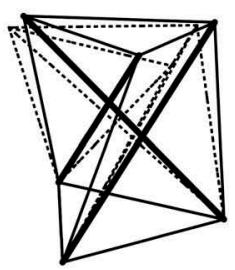

Figure 1. Simplex module: a) geometry, b) normalised self-stress state, c) infinitesimal mechanism.

Table 1. Coordinates of the nodes [m] of the single Simplex module.

\begin{tabular}{lllllll}
\hline No. of node $(i)$ & 1 & 2 & 3 & 4 & 5 & 6 \\
\hline$x$ & -0.500 & 0.000 & 0.289 & -0.577 & 0.289 & 0.289 \\
$y$ & 0.289 & -0.577 & 0.289 & 0.000 & -0.500 & 0.500 \\
$z$ & 0.000 & 0.000 & 0.000 & 1.000 & 1.000 & 1.000 \\
\hline
\end{tabular}

compatibility matrix $\mathbf{B}\left(€ \mathbb{R}^{12 \times 12}\right.$ is square, therefore the matrices $\mathbf{B B}^{T}$ and $\mathbf{B}^{T} \mathbf{B}$ are equal too. There is one zero eigenvalue in both matrices, thus one self-stress state (Figure 1b) and one mechanism is identified (Figure 1c) - summarised results of performed qualitative analyses are shown in Table 2. All eigenvalues of the matrix $\left[\mathbf{B}^{T} \mathbf{E B}+\mathbf{K}_{G}(\mathbf{S})\right]$ are positive thus the identified mechanism is infinitesimal and the stability of the structure is ensured. The model S1 meets all tensegrity features, it means that it can be classified as the ideal tensegrity.

Next, tensegrity towers built with Simplex module are analysed. Modules are linear connected in a strut-strut system. The models consisting with two modules - model S2 and six modules model S6 are considered (Kłosowska et al. 2018). The structures are supported as a model S1. For both models self-stress states $(S)$ and mechanisms $(M)$ are identified. The number of them is equal number of modules (Table 2). None of the self-stress states identifies correctly struts and cables so for the quantitative analysis superposed and normalized self-stress state for the single modified Simplex module is taken into account. All eigenvalues of the stiffness matrix $\left[\mathbf{B}^{T} \mathbf{E B}+\mathbf{K}_{G}(\mathbf{S})\right]$ are positive so towers are stable. Other than mentioned above characteristic features $S$ and $M$, the structure satisfies requirements of characteristics $T$ (it is a truss) and $C$ (tensile elements are cables and have no rigidity in compression). The analysed tensegrity towers can be classified as the structures with tensegrity features of class 1 (Obara 2019).

\subsection{Quantitative analysis}

The influence of the level of self-stress state on the normal forces (Figure 2) are analysed as the first. Next, changes in the load-bearing capacity ratio $W_{\max }$ for cables (Figure 3a) and for struts (Figure 3b), the stiffness parameter GPS (Figure 4a) and the reliability index $\beta$ (Figure $4 \mathrm{~b}$ ) are presented. The external load $P$ prestresses the structure - additional tensile and compressive forces are generated respectively in the cables and struts. After introducing the self-stress state, normal forces from the external load successively decrease. For example,

Table 2. Results of the qualitative analysis of the towers built with the Simplex modules.

\begin{tabular}{lllllll}
\hline $\begin{array}{l}\text { No. of single } \\
\text { modules }\end{array}$ & $\begin{array}{l}\text { No. } \\
\text { of } \\
\text { nodes }\end{array}$ & $\begin{array}{l}\text { No. } \\
\text { of elements }\end{array}$ & $\begin{array}{l}\text { Degrees of } \\
\text { freedom }\end{array}$ & $\begin{array}{l}\text { No. } \\
\text { of mechan- } \\
\text { isms }\end{array}$ & $\begin{array}{l}\text { No. of } \\
\text { self-stress } \\
\text { states }\end{array}$ & Classification \\
\hline 1 & 6 & 12 & 12 & 1 & 1 & ideal tensegrity \\
\hline 2 & 9 & 21 & 21 & 2 & 2 & structures with tensegrity \\
6 & 21 & 56 & 56 & 6 & 6 & features of class 1 \\
\hline
\end{tabular}


in Figure 2 presented a change in the value of normal forces arising from loads $N(P)$ and normal forces generated in total by the load and prestress forces $N(P+S)$. The analyses performed showed that the effect of the self-stress state is greater at lower load and that the impact of the load is most significant at low values of initial prestress forces.

The initial prestress forces affect normal forces $N$, hence on effort of structure. In Figure 3 the load-bearing capacity ratio $W_{\max }$ are presented. For the minimum level of self-stress state

a)

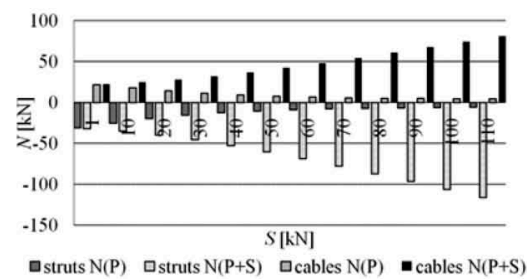

c)

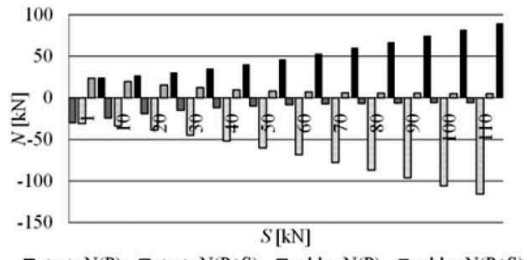

e)

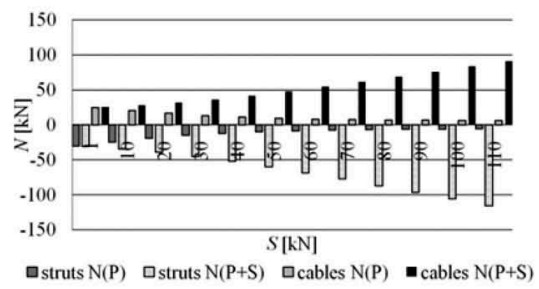

b)

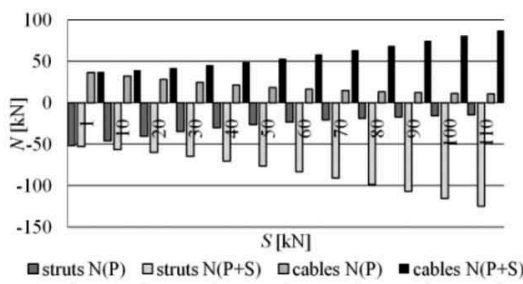

d)

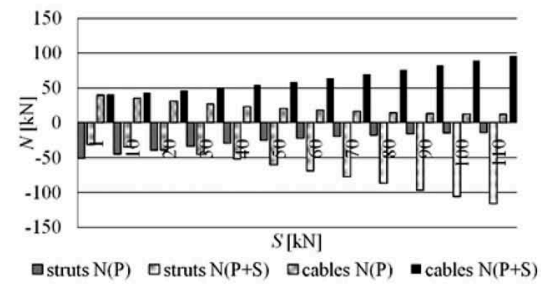

f)

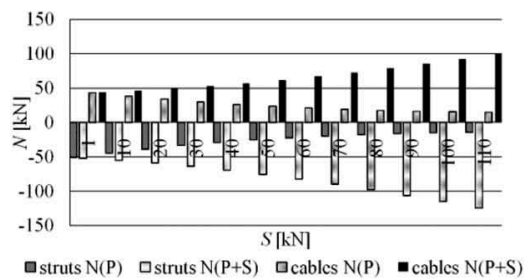

Figure 2. Influence of the self-stress state on the normal forces: a) $P=-10 \mathrm{kN}$ for model $\mathrm{S} 1, \mathrm{~b}$ ) $P=-20 \mathrm{kN}$ for model S1, c) $P=-10 \mathrm{kN}$ for model S2, d) $P=-20 \mathrm{kN}$ for model S2, e) $P=-10 \mathrm{kN}$ for model S6, f) $P=-20 \mathrm{kN}$ for model S6.

a)

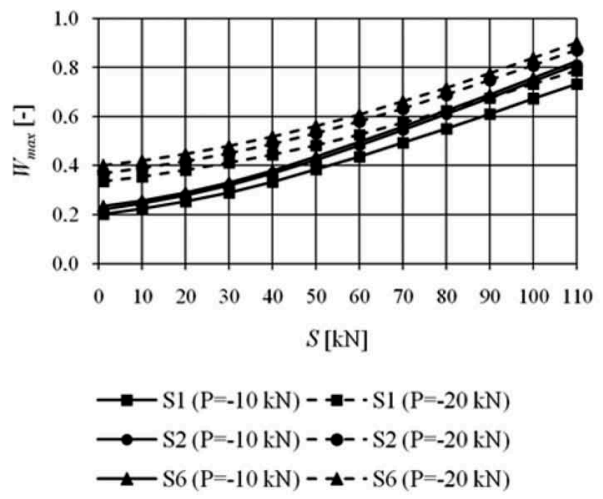

b)

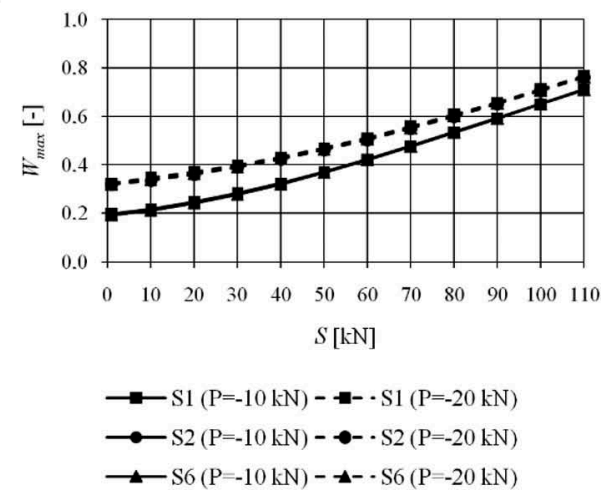

Figure 3. Influence of the self-stress state on load-bearing capacity ratio $W_{\max }$ : a) for cables, b) for struts. 
a)

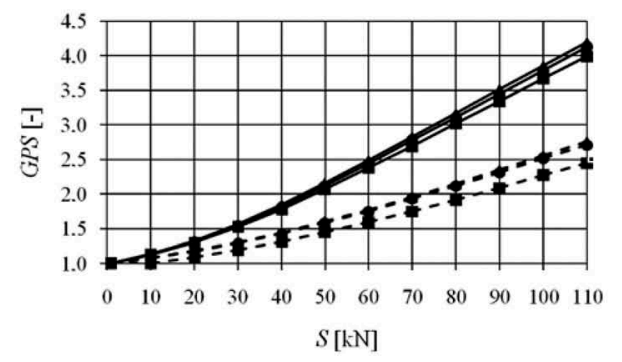

b)

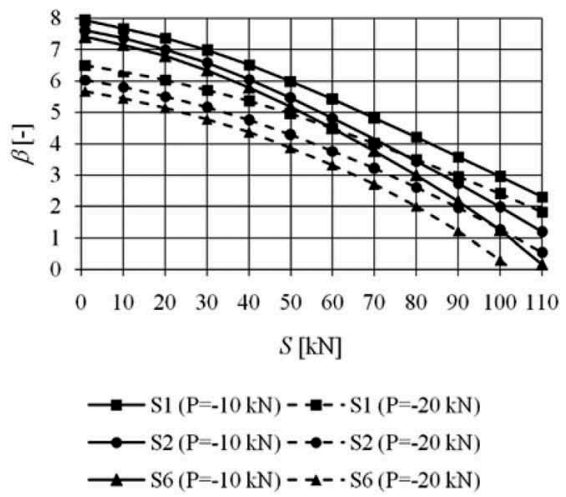

Figure 4. Influence of the self-stress state on: a) global parameter of stiffness GPS, b) reliability index $\beta$.

the increase of the applied force by $10 \mathrm{kN}$ causes the reduce of the maximum load-bearing capacity ratio by the over 60 percentage points. The impact of the load, like previous, decreasing with increasing the level of self-stress state - the same change in the level of the applied force causes the reduce of the maximum load-bearing capacity ratio by the 7-9 percentage points. In addition the effort of structure, the stiffness was monitored too. The global stiffness parameter GPS is calculated. The nature of changes in theGPS parameter is presented in Figure 4a. At maximum of the self-stress state in case $P=-10 \mathrm{kN} G P S$ is 1.51 .6 times as high as that for $P=-20 \mathrm{kN}$. This confirms the earlier conclusions that the influence of selfstress state decreases with increasing load. The initial prestress forces causes increasing the stiffness of structures, but significantly influence on decreasing the reliability index (Figure 4b) Despite to the maximum level of self-stress state does not cause the exceedance of the load-bearing capacity of the elements, the required standard level of reliability $\beta=3.8$ is exceeded.

\section{CONCLUSIONS}

In this paper, the qualitative and quantitative analyses of the towers built with the Simplex modules were performed. Analysed structures were classified as an ideal tensegrity or as a structure with tensegrity features of class 1 . In both cases, the self-stress states and infinitesimal mechanisms were identified. The stiffness of these structures depends not only on the geometry and material properties, but also on the prestress level (which stabilizes the infinitesimal mechanisms that occur) and the level of external load. External load induces additional stress in the system, and the impact of the load is most significant for the low values of the initial prestress forces. As the level of prestress increases, the effect of the external load decreases. Moreover, the initial prestress forces have a greater influence on the total stiffness of the structure with smaller external loads. Similarly, like for stiffness of tensegrity structures, in the case of effort, the influence of self-stress state decreases with increasing loads. The effort of cables is higher than that of struts and the difference between them increases with the increment of the prestress level.

Generally, with increasing the initial prestress forces the stiffness of structures increase. Unfortunately, the self-stress state significantly influence on decreasing the reliability index. Despite to the maximum level of self-stress state does not cause the exceedance of the load-bearing capacity of the elements, the required standard level of is exceeded. Therefore, in the case of tensegrity structures checking only the load-bearing capacity ratio is insufficient. 


\section{REFERENCES}

Emmerich, D.G. Construction de Reseaux Autotendants. French Patent 1,377,290, 28 September 1964.

Fuller, R.B. Tensile-integrity structures. U S Patent No 3.063.521, 1962.

Gilewski, W. et al. 2015. Application of singular value decomposition for qualitative analysis of truss and tensegrity structures. Acta Sci. Pol. Hortorum Cultus 14 (3): 14.

Gilewski, W. et al. 2019. Parametric analysis of some tensegrity structures. In Proceedings of MATEC Web of Conferences: 262, 10003.

Kłosowska, J. et al. 2018. Self-stress control of real civil engineering tensegrity structures. In AIP Conference Proceedings: 1922 (1), 150004.

Mochocki, W et al. 2018. Influence of Truss Topology on Reliability Index. IOP Conference Series: Materials Science and Engineering, 471.

Mochocki, W et al. 2019. Analysis of basic failure scenarios of a truss tower in a probabilistic approach. Applied Sciences-Basel 9 (13), 2662.

Mochocki, W et al. 2020. Impact of the wind load probability distribution and connection types on the reliability index of truss towers. Journal of Theoretical and Applied Mechanics 58 (2): 403-414.

Obara, P. 2019. Dynamic and dynamic stability of tensegrity structures (in Polish). Kielce: Wydawnictwo Politechniki Świętokrzyskiej.

Obara, P. \& Tomasik, J. 2020. Parametric analysis of tensegrity plate-like structures: Part 1 - qualitative analysis. Applied Sciences 10: 7042.

Pellegrino, S. 1993. Structural computations with the singular value decomposition of the equilibrium matrix. International Journal of Solids and Structures 30 (21): 3025-3035.

PN-EN 1990:2004. Eurocode: Basis of Structural Design; PKN: Warszawa, Poland, 2004.

Schlaich, M. 2004. The Messeturm in Rostock. A Tensegrity Tower. Journal of the International Association for Shell and Spatial Structures IASS 45 (2), 145: 93-98.

Snelson, K. Continuous tension, discontinuous compression structures. U S Patent No 3,169,611, 1965.

Snelson, K. 2013. Art and Ideas. NY: Kenneth Snelson In Association With Marlborough Gallery.

Zienkiewicz, O.C. \& Taylor, R.L. 2000. The Finite Element Method. Vol. 1. The Basis. London: Elsevier Butterworth-Heinemann. 